\title{
FEMINISMO POSCOLONIAL Y HEGEMONÍA OCCIDENTAL: UNA DECONSTRUCCIÓN EPISTEMOLÓGICA
}

\author{
POSCOLONIAL FEMINISM AND WESTERN HEGEMONY: \\ AN EPISTEMOLOGICAL DECONSTRUCTION
}

Daniel Peres Díaz

Universidad de Granada

\section{RESUMEN}

El objetivo del presente artículo consiste en presentar las líneas maestras del feminismo poscolonial, el cual se articula hoy como una alternativa teórica de carácter crítico al feminismo hegemónico o etnocéntrico. Se analizan los principales avances conceptuales y epistemológicos que han cristalizado en dicho feminismo poscolonial, con especial atención en el feminismo africano. La crítica a la lógica bivalente, al etnocentrismo metodológico y al espíritu colonialista de la Modernidad ilustrada europea vertebra la exposición sistemática que se hace de las diversas propuestas nacidas de los «feminismos periféricos». En última instancia, se propone una resignificación de las categorías fundamentales del pensamiento feminista, bosquejándose así una visión alternativa de la política, los Derechos Humanos y la emancipación social.

Palabras clave: feminismo, colonialismo, poscolonialismo, poder, género, raza.

\section{ABSTRACT}

The aim of this article is to present the main lines of postcolonial feminism, which is articulated today as a theoretical critical alternative to the hegemonic or ethnocentric feminism. We analyze the main conceptual and epistemological advances that have crystallized in this postcolonial feminism, paying particular attention to African feminism. Criticism of bivalent logic, methodological ethnocentrism and colonialist spirit of the European Enlightenment Modernity articulates the systematic exposition of the various proposals derived from the «peripheral feminisms». Ultimately, a redefinition of the fundamental categories of feminist thought is proposed, while broadly outlined an alternative view of politics, human rights and social emancipation.

Keywords: Feminism, Colonialism, Post-Colonialism, Power, Gender, Race. 


\section{Introducción}

¿Qué es el feminismo o los feminismos? ¿Qué se entiende por feminismo fuera de Occidente? ¿Qué significa que el feminismo occidental se ha convertido en hegemónico? ¿Qué implicaciones tiene para el feminismo la lucha de las mujeres desde la periferia? Estas preguntas articulan algunas ideas clave del presente artículo. Sabemos que la historia de las ideas feministas está marcada desde sus inicios por la tensión conceptual que caracteriza a todo proyecto incompleto, histórico o contingente. El hipercriticismo característico del feminismo es un arma de doble filo, ya que, si bien posibilita la emergencia de un pensamiento combativo contra las estructuras de poder, no es menos cierto que con el «exceso» de crítica se corre el peligro de debilitar la unidad en la lucha por los derechos y la emancipación de la mujer al cuestionarse incluso los fundamentos más elementales del propio feminismo.

Así, al estar el feminismo en un proceso constante de reformulación y crítica, conviene deconstruir la idea de que el feminismo es solamente uno. $Y$ es que la riqueza de feminismos es un valor en sí mismo para la propia crítica feminista. En verdad, la diversidad y pluralidad de feminismos no debe verse como un elemento negativo o limitador del potencial transformador de la lucha por la igualdad de las mujeres, sino como la consecuencia lógica de la inclusión de la crítica feminista en diversos contextos sociales, económicos, políticos, culturales, institucionales, demográficos, etc. Cabe reseñar que el valor del feminismo está en «los feminismos», esto es, en su capacidad para generar distintas respuestas ante situaciones que, de facto, son diferentes. Por lo tanto, la «unidad» del feminismo debe cifrarse en la no universalización del modelo de resistencia de las mujeres ante la opresión que sufren, pues en los diferentes lugares donde existe dicha opresión las respuestas van a variar en función del contexto.

En cualquier caso, la propia evolución de los movimientos feministas es una prueba clara de lo expuesto en el párrafo anterior. Tal y como establece la filósofa Ana de Miguel (2011), existen en la historia feminista occidental tres olas o generaciones, a saber:

a. Primera ola feminista ilustrada que se inicia con la Revolución Francesa (1789) y cuyas autoras de referencia serían Poullain de Barre, Olympe de Gouges y Mary Wollstonecraft.

b. Segunda ola sufragista, que arranca con la Declaración de Seneca Falls (1848) y que está representada por autoras como Emmeline Pankhurst o Sojourner Truth.

c. Tercera ola, que inaugura el feminismo contemporáneo y que se centra en la crítica a la idea de que existe un modelo de mujer. Las autoras más relevantes en esta tercera ola serían Simone de Beauvoir (El segundo sexo) y Betty Friedan (La mística de la feminidad). 
En el transcurso de las sucesivas olas se observa un movimiento de fractura y reconstrucción constante de los postulados feministas, movimiento que convierte al pensamiento feminista en un espacio genuino de reflexión y debate. Por ello, no es de extrañar que haya surgido en nuestros días el feminismo poscolonial, que se sitúa en la periferia del feminismo europeo (Mohanty, 2008), es decir, que critica los postulados analíticos y conceptuales del feminismo europeo u occidental desde la experiencia de las mujeres negras. En efecto, el feminismo occidental ha sido predominantemente reivindicado por mujeres blancas, de clase media-alta, que ejercen profesiones liberales en un marco de economía de mercado y que reproducen sobre determinados colectivos, a veces inconscientemente, las mismas relaciones de dominio patriarcales que buscan eliminar (Mignolo, 1995).

En este marco, alcanza forma el objetivo del presente trabajo, que consiste en presentar, tal y como se adelantaba en el resumen, las líneas maestras del feminismo poscolonial, el cual se articula hoy como una alternativa teórica de carácter crítico al feminismo hegemónico o etnocéntrico. La exposición sistemática de los feminismos poscoloniales nos va a ofrecer las condiciones de posibilidad de una crítica de las nociones fundamentales del pensamiento categorial y binario de la filosofía occidental (Medina Martín, 2014). Con ello, podremos conectar los feminismos poscoloniales con un proyecto más amplio de reconceptualización de la política, la lucha por los derechos humanos y la emancipación social de los pueblos, pues la propia crítica que los feminismos poscoloniales dirigen hacia el feminismo occidental implica una forma decolonial de entender la organización política de la comunidad.

\section{Una exposición del feminismo poscolonial}

La primera tarea que nos ocupa consiste en esbozar las líneas teóricas del «feminismo poscolonial»; tras esto, podremos diseccionar sus elementos más fundamentales y dar cuenta de los avances epistemológicos que se derivan de la crítica al feminismo occidental. Así pues, podemos decir que:

Los «feminismos poscoloniales» son movimientos político-sociales complejos y dinámicos que pretenden transformar las relaciones asimétricas de opresión entre los sexos, a partir del cuestionamiento de categorías, conceptos e ideas en relación al género, con la finalidad de proponer nuevos significados que consideren las experiencias de mujeres provenientes de realidades invisibilizadas (Ron Erráez, 2014: 40).

Desde esta perspectiva, se entiende que la opresión ejercida sobre la mujer por las estructuras del poder fluctúa en virtud del contexto social y cultural, de suerte que las 
eventuales soluciones institucionales, políticas, económicas, educativas y de otra índole a esa discriminación no pueden, en modo algo, ser universalizables; es decir, no pueden exportarse fuera de cada uno de esos marcos concretos. En la medida en que el feminismo hegemónico ha intentado universalizar su análisis, estaría operando como otra fuerza más de discriminación hacia aquellas mujeres que habitan en contextos de marginación no representables en los términos del pensamiento filosófico feminista occidental'.

Conviene aclarar que el término «poscolonial» no ha de entenderse aquí exclusivamente como la etapa política e histórica que sucede al proceso de descolonización, sino también como un modo de narración -un relato- que cifra su núcleo teórico en el cuestionamiento de la herencia colonizadora, de sus experiencias y sus saberes. El colonialismo es, desde esta perspectiva, un conjunto de corrientes teóricas y analíticas, firmemente enraizadas, que penetran en el sustrato cultural y que tienen como rasgo común el otorgar primacía a las relaciones desiguales entre el Norte y el Sur en la explicación del mundo contemporáneo (Sousa Santos, 2006).

La clave está en comprender que el fin del colonialismo no implica necesariamente el fin de la colonialidad del poder (Quijano, 2005), toda vez que la ausencia de un poder colonial «macropolítico» a nivel estatal no tiene su correlato material directo en la eliminación de todas las formas de discriminación, a veces más difusas, subrepticias, incardinadas en eso que Foucault (1976) llamaba «microfísica del poder»². A este respecto, las palabras de Sousa Santos son esclarecedoras:

La dificultad de imaginar la alternativa al colonialismo reside en que el colonialismo no es solo ni principalmente una política de Estado, como sucedía durante el colonialismo de ocupación extranjera; es una gramática social muy vasta que atraviesa la sociabilidad, el espacio público y el espacio privado, la cultura, las mentalidades y las subjetividades. Es, en resumen, un modo

\footnotetext{
1 Nos es imposible tratar aquí, por razones de brevedad, el surgimiento de las nuevas teorías críticas en oposición al feminismo tradicional en el seno de los países occidentales, sobre todo en los Estados Unidos. Algunas propuestas como la teoría queer, el ciberfeminismo, el transfeminismo, el feminismo de la diferencia, el feminismo postestructural y el movimiento Girlie, entre otras, hacen un énfasis explícito en la idea de que no existe un único modelo de mujer. De este modo, atacan la metafísica esencialista tradicional en Occidente al proponer, bien desde una propuesta constructivista/culturalista, bien desde una propuesta de transformación científicotecnológica del cuerpo, un sujeto nómada, un Cyborg o híbrido que ya no sería una «mujer» en el sentido estricto de la palabra, sino una «alteridad» (Peres Díaz, 2016). Dada la enorme profusión de literatura en relación a este enfoque, nos limitamos a hacer una breve referencia para dejar claro que la crítica al feminismo tradicional no es patrimonio exclusivo de los feminismos poscoloniales, si bien su invisibilización académica constituye un motivo suficientemente relevante en términos metodológicos como para ocupar el centro de la reflexión de este artículo.

2 No podemos entrar a analizar en profundidad los vínculos entre la propuesta de Quijano (2005) y la de Foucault (1976). Sí cabe decir, no obstante, que ambos autores comparten una concepción no orgánica y dispersa del poder, «biopolítica», en la medida en que tanto para Quijano como para Foucault el fin del colonialismo no implican el fin del racismo instaurado en la "gramática» de gobernabilidad de los países colonizados. Así, el poder, según esta perspectiva, se trasmite mediante dispositivos de control y vigilancia, dispositivos que se materializan en una colonialidad institucional que no es visiblemente macropolítica o estructural.
} 
de vivir y convivir muchas veces compartido por quienes se benefician de él y por los que lo sufren (Sousa Santos, 2010: 15).

Por lo tanto, no basta con la emancipación política de un país cualquiera, a priori alcanzable mediante la liberación nacional y la constitución de un pueblo soberano, sino que es necesario, en paralelo, cuestionar y deconstruir el imaginario, el relato, esto es, los conceptos legados por los países colonizadores. Ese imaginario se «dispersa» mediante prácticas sociales cotidianas, que reproducen una hegemonía cultural que no es fácilmente identificable (López Souto, 1999). Así, la perspectiva poscolonial «parte de la idea de que a partir de los márgenes o de las periferias, las estructuras de poder y de saber son más visibles» (Sousa Santos, 2006: 39).

En términos generales, las aproximaciones poscoloniales lo que buscan es reinterpretar al sujeto y la historia coloniales, tal y como estos han sido representados tradicionalmente por Occidente. Así, «sus distintos autores tratan de explicitar la relación entre la formación de conocimiento sobre las colonias y excolonias, el ejercicio de poder hacia ellas, y los distintos tipos de interpenetración que existen entre sociedades colonizadas y colonizadoras» (Arreaza y Tickner, 2002: 22-23). En consecuencia, un análisis poscolonial tiene como objetivo último lograr que el sujeto colonizado se convierta en un sujeto genuino con capacidad de ser agente de su propio destino.

Profundizando un poco más en esa distinción entre colonialismo y colonialidad, podemos afirmar que el primero remite a un sistema de dominación político-administrativo correspondiente a determinados períodos históricos y lugares concretos donde se ejerció un dominio imperial. En cuanto a la colonialidad, es una:

(...) categoría que debemos a Aníbal Quijano, [y] remite a la estructura de dominio subyacente al control ejercido durante la colonización española y lusitana; que permanece y se extiende - en múltiples dimensiones y regiones - una vez acabada ésta. Esta distinción es nodal ya que nos permite distinguir que el pensamiento poscolonial opera en el marco de problematización abierto por el colonialismo (Ivanna Gigena, 2009: 5).

Por todo ello, algunos autores como Pérez Flores (2014) entienden que lo «poscolonial es postestructural, [pues] cuestiona ya la lista de categorías, deconstruye el edificio colonial desde los cimientos. Y es al tiempo edificación, proliferación de identidades transcategoriales, creación semiótica de un universo poshumanista» (Pérez Flores, 2014: 112). He aquí la crítica al binarismo propio del pensamiento occidental como un pilar fundamental de la teoría poscolonial, en general, y del pensamiento feminista poscolonial, en particular. Tendremos ocasión de analizar esta idea del binarismo con más profundidad en el apartado tercero. 
Así, podemos plantear la hipótesis de que el «patriarcado» no es una estructura social, política y económica común, sino una realidad que se adapta y modula según diversas circunstancias y condiciones culturales. De este modo, si hay distintos patriarcados (Rodríguez Martínez, 2011), entonces ha de haber, por fuerza, distintos «modos de resistencia», es decir, distintos modos de respuesta.

Si los significados se entretejen en contextos culturales diversos, se infiere entonces que es totalmente inútil el intento de pretender «emancipar» a la mujer con base en una estrategia con pretensión de validez universal. Es decir, el feminismo poscolonial parte de la premisa de que no hay un único sistema patriarcal o un único modelo de racionalidad, por lo que «este tipo de feminismos se orienta a visibilizar las realidades, conocimientos y experiencias de las mujeres de contextos no occidentales, con objeto de que estas sean reconocidas como productoras de racionalidades y fuentes de reivindicación de derechos» (Ron Erráez, 2014: 43).

El caso es que las diferentes perspectivas del pensamiento feminista clásico - liberal, marxista-socialista, psicoanalítico - giraban en torno al análisis crítico sobre las relaciones entre hombres y mujeres en las familias de clase media nucleares, marginando de esta manera aquellos lugares en los cuales la industrialización no había impactado con un mismo grado de intensidad y dejando al margen a las mujeres de las clases sociales más bajas (Rodríguez Martínez, 2011; Medina Martín, 2013, 2014). Por eso, estas autoras hablan del feminismo poscolonial en términos de "feminismos periféricos», ya que entienden que estos nuevos feminismos vienen marcados por la descontextualización geográfica y cultural del propio análisis feminista, lo que significa que todo feminismo poscolonial es tal porque no quiere ni pretende generalizar dicho análisis.

En consecuencia, no se puede entender el feminismo como una categoría analítica con validez universal (Rodríguez Martínez, 201 1; Medina Martín, 2013, 2014; Ron Erráez, 2014), pues detrás de cada teoría y práctica feminista hay una génesis y una evolución histórica, sociopolítica, económica, cultural, institucional y simbólica determinada. Y esa génesis y evolución dan lugar a realidades singulares, es decir, no subsumibles bajo esquemas universalistas. No es lo mismo, obvia decirlo, vivir en una economía postindustrial que en una economía agrícola; en una sociedad de fuerte diferenciación étnica y lingüística que en una sociedad relativamente homogénea; o en país democrático que en país autoritario.

Sea como fuere, la genealogía que emprenden los feminismos periféricos viene marcada por la idea de que se debe superar una lectura de las subordinaciones de las mujeres que solamente se acota a la diferencia sexual. En este sentido, las lógicas clásicas de las luchas feministas se superponen con otras lógicas de diversa índole, y lejos de 
tener que optar entre luchas identitarias culturales y luchas feministas, o entre luchas por la redistribución o el reconocimiento, «los feminismos poscoloniales o periféricos exigen conjuntamente reconocimiento y redistribución tanto en lo global como hacia dentro de sus propias colectividades identitarias» (Medina Martín, 2014: 93), apostando de este modo por la variedad de identidades en la estela de una «epistemología de la frontera» (Rodríguez Ortiz, 2014).

En este marco, autoras como Chandra Mohanty (2008) u Oyeronke Oyewumi (2002) han acuñado las expresiones «feminismo del tercer mundo» y «feminismo africano» respectivamente para hacer referencia a ese feminismo poscolonial practicado desde la periferia y que incorpora la experiencia de los países colonizados, víctimas del capitalismo y las sucesivas guerras de dominación.

En particular, el análisis crítico-discursivo de Mohanty puede ofrecer algunas coordenadas válidas para que el lector o lectora entienda de un modo genuino el espacio epistemológico en el que se sitúa el feminismo poscolonial. A pesar de las dificultades de entender «Occidente» como un bloque monolítico, compacto y homogéneo, y sin perjuicio de las excepciones que pudieran darse, los análisis feministas de la situación de la mujer realizados en y desde Occidente presuponen toda una serie de representaciones colectivas cuyos efectos políticos son innegables, en la medida en que ese modelo teórico construido sobre la base histórica de Occidente extrapola un modo de entender a la mujer como sujeto y categoría política, y afectan, por tanto, a la manera de organizar la convivencia y la comunidad política.

A partir de esta idea fuerza, Mohanty (2008) deconstruye las tres premisas analíticas fundamentales del feminismo occidental. La primera presuposición analítica -señala la autora- involucra la ubicación estratégica de la categoría «mujeres» insertada en el contexto de análisis. El feminismo occidental parte de la presuposición de que la categoría «mujeres» responde a un grupo «ya constituido y coherente, con intereses y deseos idénticos sin importar la clase social, la ubicación o las contradicciones raciales o étnicas» (Mohanty, 2008: 5), lo que implica una noción de diferencia sexual o de género, o incluso una noción de patriarcado, que puede aplicarse de forma universal y a todas las culturas. En términos concisos, el feminismo occidental es un feminismo universalista y con aspiración de validez ahistórica.

La segunda presuposición analítica, dice Mohanty (2008: 5), se refiere a que es «evidente a nivel metodológico, en la carencia crítica con la que se presenta la 'evidencia' que sustenta la universalidad y la validez para todas las culturas». Estaríamos, pues, ante un segundo nivel, que sobrepasa el plano conceptual y se adentra en el metodológico, esto es, en 
las formas concretas de investigación, teórica y empírica, que en último término dan soporte a las publicaciones, conferencias y prácticas sociales feministas en el mundo occidental.

Y la tercera presuposición analítica tiene que ver con el modelo de poder y lucha que sugieren e implican los dos presupuestos anteriores, de suerte que la noción de mujer oprimida que se maneja es homogénea y se aplica al grupo, dando lugar, también, a una «mujer promedio del tercer mundo» que atrofia la singularidad específica de cada contexto geográfico y cultural.

Así pues, deconstruyendo estos tres presupuestos analíticos, Mohanty (2008) concluye que «conceptos tales como la reproducción, la división sexual del trabajo, la familia, el matrimonio, el hogar, el patriarcado, etc. se usan a menudo sin especificarlos en contextos culturales e históricos locales» (Mohanty, 2008: 13-14). Este enfoque universalista y etnocéntrico estructura una dicotomía entre hombres y mujeres, los «preconstituye» o prefigura como grupos dotados de coherencia, de tal modo que los intereses de esos grupos les son dados con anterioridad. Así, el patriarcado adquiere una significación hegemónica en todo contexto, denotando el simple y burdo dominio del hombre sobre la mujer, lo que impide una genuina comprensión de los fenómenos complejos de países no occidentales, en los cuales se interrelacionan e imbrican elementos que a su vez engloban conocimientos, experiencias y marcos simbólicos muy dispares más allá de la simple variable de género.

A fin de cuentas, hay una construcción de la «otredad» que es superficial y poco precisa, entendiendo por la misma la operación de subsumir bajo categorías con aspiración de validez universal realidades culturales complejas no reductibles a una arquitectura conceptual maximalista. Frente a esta visión universalista, propia de la Modernidad ilustrada y de claro corte colonial, conviene revitalizar un enfoque interseccional, es decir, un enfoque que se haga cargo de los puntos de conexión entre los conceptos de raza, clase, género y sexualidad para denunciar, con ello, las diferentes fisonomías de la pobreza y de la violencia a la que se ven sometidas las mujeres negras, víctimas de un patriarcado de diferente naturaleza y caracterizado por el inseparable vínculo entre colonialidad del poder y género (Lugones, 2008).

La interseccionalidad, como uno de los conceptos fundamentales en la articulación del feminismo poscolonial, debe ponerse en relación con la propia idea de la colonialidad del poder. $Y$ es que, frente al análisis de género, el enfoque interseccional surge como consecuencia de la superposición de exclusiones de matriz colonial. A este respecto, una genealogía interesante del concepto de colonialidad del género es la que da Lugones (2011), la cual, a pesar de su extensión, no puedo evitar reproducir:

164

Dossiers Feministes, 22, 2017, 157-177 - ISSN: $1139-1219$ - DOI: http://dx.doi.org/10.6035/Dossiers.2017.22.10 
Concibo la jerarquía dicotómica entre lo humano y lo no humano como la dicotomía central de la modernidad colonial. Comenzando con la colonización de las Américas y del Caribe, se impuso una distinción dicotómica, jerárquica entre humano y no humano sobre los colonizados al servicio del hombre occidental. (...) Los pueblos indígenas de las Américas y los africanos esclavizados se clasificaban como no humanos, como animales, incontrolablemente sexuales y salvajes. El hombre moderno europeo, burgués, colonial, se convirtió en sujeto/agente, apto para gobernar, para la vida pública, un ser de civilización, heterosexual, cristiano, un ser de mente y razón. La mujer europea burguesa no era entendida como su complemento, sino como alguien que reproducía la raza y el capital mediante su pureza sexual, su pasividad, y su atadura al hogar en servicio al hombre blanco europeo burgués. La imposición de estas categorías dicotómicas quedó entretejida con la historicidad de las relaciones, incluyendo las relaciones íntimas (Lugones, 2011: 106).

Es decir, la colonialidad del poder tiene su raíz en una organización total de la vida de los sujetos colonizados. Por eso, tal y como afirma Lugones en la cita, la mujer europea era entendida como parte de ese sistema completo y de explotación y opresión. El hecho de que las relaciones dicotómicas penetraran hasta la profundidad de las relaciones íntimas conduce a la idea de que la deconstrucción de toda esa matriz colonial es el paso previo a la emancipación de la mujer. De ahí la necesidad del enfoque interseccional.

Se puede, así, conectar el feminismo poscolonial - como idea articuladora del presente artículo - con las prácticas políticas y sociales de la lucha antirracista. Esta convergencia se expresa de un modo claro en el Black Feminism y el feminismo chicano de los Estados Unidos, así como en todos los movimientos feministas indígenas de América Latina y el Caribe (Curiel, 2007), asumiendo que descolonizar supone registrar producciones teóricas y prácticas subalternizadas, racializadas, sexualizadas, por lo que es importante reconocer a tantas mujeres cuyas luchas sirvieron de antecedente para construir luego estas teorías. Es decir, el feminismo poscolonial, como teoría, bebe directamente de prácticas antirracistas claramente políticas y reivindicativas de derechos.

Se suele situar como un antecedente importantísimo del Black Feminism el texto Mujeres, raza y clase (2005), de Angela Davis, autora que realiza un recorrido histórico por las diferentes implicaciones y singladuras del movimiento antiesclavista, poniendo el énfasis central en el racismo presente en el «movimiento sufragista de las mujeres ${ }^{3}$, la violación y el racismo bajo el mito del violador negro o el trabajo doméstico de las mujeres negras, todo ello, en los análisis feministas de las mujeres negras sobre una nueva feminidad» (Medina Martín, 2013: 65).

3 No podemos entrar a analizar en profundidad este punto, pero sí decir que, para Angela Davis (2005), el movimiento sufragista es racista porque arroja a la mujer negra a una nueva forma de esclavitud, a saber, aquella en la que el hombre negro tiene derecho al voto pero no se garantiza los derechos individuales de la mujer negra. Como dice Angela Davis (2005), citando irónicamente a Elizabeth Cady Stanton, «es mejor ser esclava de un hombre blanco educado que de un negro degradado e ignorante» (p. 77). 
Con base en ello, urge la necesidad de modificar los discursos feministas en los centros de producción de conocimiento y saber en el Norte global. El objetivo ha de ser la visibilización académica de otros enfoques, metodologías y contextos de estudio. Puesto que:

Si los feminismos occidentales no aceptan que han reproducido diferentes formas de «racismo/ sexismo epistémico», no habrá descolonización de los feminismos (entiéndase, eliminación de cualquier tipo de jerarquía donde unas mujeres decidan qué es lo mejor para el resto de las mujeres), no será posible emitir valoraciones desprejuiciadas sobre los aportes en términos emancipatorios hechos por las mujeres desde posiciones distintas a la línea ilustrada (Limic, 2015: 153).

Es imprescindible, asimismo, realizar una reflexión crítica sobre la deslocalización de las epistemologías y metodologías feministas, que han sido históricamente invisibilizadas, y de su conveniencia o no de importarlas, usarlas y reapropiarlas en otros contextos. La experiencia demuestra que cuando se intenta representar la totalidad de un grupo y se inscriben desde posiciones de privilegio "generan un 'equívoco' que es el del discurso universalizador. Este equívoco puede producir $y$, de hecho produce, una violencia conocida desde hace siglos, que es la violencia colonial» (Ruiz Trejo y Betemps, 2015: 179).

Así las cosas, una vez expuesto en términos muy generales el marco epistémico en el que opera el feminismo poscolonial, podemos emprender algunas matizaciones centrándonos en las particularidades del feminismo africano como subtipo de feminismo poscolonial.

\section{Breve excursus al feminismo africano}

En consonancia con lo expuesto en el apartado anterior, y acotando el espacio al continente africano, autoras como Oyewumi (2002) problematizan sobre las dificultades de las «epistemologías africanas» para conceptualizar el género desde las coordenadas ofrecidas por el feminismo occidental. Este punto de partida nos puede servir para emprender un breve excursus al feminismo africano como paradigma de feminismo poscolonial, toda vez que su eje vertebrador es la incapacidad de asumir las categorías binarias del pensamiento feminista occidental por ser inservibles para explicar en términos adecuados las dinámicas emancipatorias del continente.

El caso es que Oyewumi (2002) va a criticar el binarismo inherente al pensamiento occidental que, sobre el presupuesto del cuerpo como dato evidente, establece dicotomías tales como hombre/mujer, masculino/femenino, biología/cultura, bien/mal, etc. Estas dicotomías son ajenas a muchas realidades africanas, en algunos casos inconmensurables,

166

Dossiers Feministes, 22, 2017, 157-177 - ISSN: $1139-1219$ - DOI: http://dx.doi.org/10.6035/Dossiers.2017.22.10 
por lo que no será posible articular estrategias de emancipación prescindiendo de los estudios antropológicos, sociológicos y culturales de muchas regiones que, en el fondo, son prácticamente desconocidas para el mundo occidental. Sin tener en cuenta la cultura como elemento modulador de las prácticas sociales, toda construcción discursiva no será más que un simple refrito ideológico de una corriente dominante (Ros, 2002).

Así las cosas, hay que advertir que el término «feminismo africano» no está exento de controversia, ya que su empleo es «tanto insuficiente para reflejar la complejidad de la realidad que pretende describir como contradictorio, en la medida en que supone definir a quien ha manifestado su deseo de autonombrarse y autodefinirse» (Zirion Landaluze e Idagarra Espel, 2015: 41). En todo caso, el punto de partida conceptual y epistemológico del feminismo africano es su heterogeneidad, pues los feminismos africanos son un fenómeno trasnacional, desarrollado en África pero también en otros lugares, a causa de las migraciones y de los movimientos forzosos (Zirion Landaluze e Idagarra Espel, 2015: 42). Ello hace que en ocasiones el feminismo africano pueda verse identificado bajo la rúbrica de «diaspórico»; en otras ocasiones, el término empleado es «feminismo negro» (Angela Davis, 2005; bell hooks, 2004).

A efectos de delimitación cronológica, habría que situar el origen del feminismo africano como tal en el año 1981, cuando la sierraleonesa Filomena Chioma Steady (1981) ${ }^{4}$ establece una relación entre los feminismos africanos, de un lado, y la autonomía y la cooperación femeninas, del otro. Así, podemos entender el feminismo africano como aquel que enfatiza las discriminaciones que sufren las mujeres africanas en virtud no solo del sexo/ género, sino también, y sobre todo, de la esclavitud, del colonialismo y del racismo (Zirion Landaluze e Idagarra Espel, 2015: 43).

Años más tarde, en 1994, Molara Ogundipe-Leslie introduce el término stiwanism (acrónimo de Social Transformations Including Women in Africa) para hacer hincapié en una propuesta de creación de una sociedad armoniosa. Para ello, es necesario, según esta óptica, que la transformación venga de la colaboración, prescindiendo de la oposición entre hombres y mujeres, lo que implica que la mujer no debe renunciar a su maternidad, ni centrarse únicamente en las cuestiones sexuales, sino, al contrario, incidir en la intersección de las dimensiones de su marginación, como son la pobreza, la falta de educación o la

\footnotetext{
4 Existe una larga tradición de resistencia de las mujeres en África que no está lo suficientemente tematizada y documentada. Por supuesto, el marco temporal elegido en este artículo es ficticio en la medida de que es imposible establecer una fecha determinada. Con todo, se elige la obra de Steady por situar un mínimo antecedente que posibilite el análisis de las manifestaciones contemporáneas del feminismo africano. No se pretende en este apartado llegar a un análisis exhaustivo de todo el feminismo africano, su origen, su evolución y, en definitiva, estudio histórico; un trabajo así requeriría de una extensión que desborda por mucho los límites del presente artículo.
} 
ausencia de horizontes. A este respecto, se enfatiza la necesidad de desarticular las causas que impiden la participación de las mujeres en la transformación social, a saber, la raza, la opresión exterior, los roles neocoloniales, el imaginario colectivo y las dificultades económicas (Ogundipe-Leslie, 1994).

A partir de ahí, llegan a nuestros días las obras de otras autoras -Oyewumi (2002), Mekgwe (2006), Nnaemeka (2004), etc.- que, conscientes del peso de los legados coloniales, reflexionan sobre cómo esa herencia ha influido en la configuración del pensamiento feminista africano. La conclusión a la que llegan es que conviene deconstruir el pensamiento feminista occidental y analizar las relaciones de poder, superando con ello la «trampa colonial» que implica estar constantemente definiéndose como la «otra» no occidental (Mekgwe, 2006).

De este modo, se asume una perspectiva interseccional en la que el género se cruza con la clase, la raza, la etnia, en un ejercicio de deconstrucción epistémica de mayor alcance, que implica un ejercicio de autodenominación -Politics of naming- para visibilizar un conocimiento «diferentemente situado» y poner rostro a la riqueza interna del debate feminista africano de cara a la consolidación de nuevas subjetividades no hegemónicas (Nnaemeka, 2004).

En síntesis, nos encontramos ante diferentes y nuevas corrientes feministas, las cuales van tomando nombre, a saber, womanism, african womanism, stiwanism, motherism y nego-feminism ${ }^{5}$, entre muchas otras. Su conjunto dibuja una cartografía de los feminismos africanos plural y diversa que comparte la convicción de que estos deben basarse en las especificidades africanas (Zirion Landaluze e Idagarra Espel, 2015: 51). El motherism, por ejemplo, ofrece una alternativa al feminismo occidental a partir de la construcción de un modo de vida basado en los valores centrales de la forma de vida africana, y centrados en la maternidad y la experiencia femenina africana (Obianuju Acholonu, 1995). Así las cosas, la corriente motherism considera como fundamental la maternidad, que es la matriz de la existencia y de la participación de la mujer en la vida comunitaria, al tiempo que critica el rechazo del feminismo occidental al rol reproductivo; tanto es así que rechaza el uso del término patriarcado, y no aborda las desigualdades en clave de género, sino en términos de colonización (Obianuju Acholonu, 1995). Es decir, se entiende que las desigualdades entre hombres y mujeres en las sociedades africanas no existían con anterioridad al proceso colonizador, por lo que la causa de la discriminación de las mujeres no se debe

5 Hemos dado algunas características de la corriente womanism y motherism, pero no de las otras citadas. Así, cabe destacar que el nego-feminism se basa, fundamentalmente en la negociación y la cooperación, mientras que la corriente stiwanism enfatiza la participación de las mujeres africanas y tiene como objetivo el rechazo de la imitación del feminismo occidental. Por su parte, la corriente african womanism se centra en el abordaje de las especificidades africanas en relación a la maternidad como eje central 168 (Zirion Landaluze e Idagarra Espel, 2015). 
a la articulación de un sistema que diferencia sexos, sino a la imposición de un modelo de dominio por parte del mundo occidental.

Pero no es exactamente lo mismo el feminismo africano que el feminismo negro, en la medida en que este último hace referencia a la lucha de las mujeres negras en países industrializados y occidentales, y cuyo máximo exponente es bell hooks (2004), quien pone de relieve la ligazón entre género y raza (Jabardo Velasco, 2008). Ilustrativa del feminismo negro es la siguiente cita de Avtar Brah (1992):

En la medida en que 'mujeres negras' conformaba una categoría altamente diferenciada en términos de clase, etnicidad y religión, e incluía a mujeres que habían migrado desde África, el subcontiente asiático y el Caribe tanto como a nacidas en Inglaterra, lo negro en el 'feminismo negro' implicaba una multiplicidad de la experiencia a la par que articulaba una posición de un sujeto feminista particular. Más aún, al poner en un primer plano una amplia gama de experiencias diaspóricas tanto en su especificidad local como global, el feminismo negro representaba la vida negra en toda su amplitud, creatividad y complejidad (Brah, 1992: 134).

En este marco, un proceso emancipatorio que quisiera imprimir desde el plano educativo una política más inclusiva e igualitaria debería, al menos, tomar como referencia los siguientes cuatro ejes de articulación (Martínez Martín, 2015: 165):

a. Entendimiento e inclusión de las diferencias.

b. La interseccionalidad con otras formas de desigualdad de clase, etnia, formación o ubicación geográfica.

c. La comprensión de saberes contra-hegemónicos y diversificación de saberes, experiencias y poderes de las mujeres africanas frente a la imposición de lógicas dominantes.

d. La ruptura de estereotipos donde las mujeres africanas son sujetos pasivos sin protagonismo en su desarrollo.

Por todo lo dicho, y sin ánimo ni mucho menos de alcanzar una caracterización exhaustiva, puede observarse en esta cartografía del feminismo africano su carácter bifronte. Por un lado, se presenta como un movimiento revitalizador del discurso feminista a escala mundial, toda vez que abre el marco epistemológico e incorpora nuevos procesos sociales, políticos e históricos al objeto de estudio del feminismo; por el otro, se constituye como un elemento de crítica sin paliativos de muchos de los presupuestos del feminismo occidental. En tanto que cara visible del feminismo poscolonial, el feminismo africano abre un surco que nos obliga a replantear algunas categorías relativas al pensamiento político y la lucha por la emancipación y los derechos humanos. 


\section{Hacia un cambio en la hegemonía cultural}

Hemos visto cómo el feminismo poscolonial deconstruye gran parte de los presupuestos epistemológicos del feminismo occidental, combatiendo el pensamiento binario y oposicional, al tiempo que pone de relieve la necesidad de repensar categorías sociopolíticas fundamentales e ir más allá de un análisis de género. En este sentido, el feminismo poscolonial implica un cambio de tendencia en la hegemonía global, esto es, supone una reconfiguración a escala planetaria de diferentes dimensiones de la organización humana como son la política, la economía, la sociedad, la reproducción, la ecología, la paz y otro sinfín de cuestiones de importante relevancia. Esa hegemonía es, ante todo, cultural, pues impone un «modo correcto» de entender los conceptos que vertebran las sociedades y que, en todo sistema político, son disputados por los diferentes grupos que integran esas sociedades.

En este contexto, la hegemonía debe ser entendida en términos de la capacidad que tienen ciertos sectores -élites mundiales y nacionales- para imponer la interpretación correcta de los conceptos que están en disputa. Por lo tanto, la lucha por la emancipación social ${ }^{6}$ deberá desplegar sus esfuerzos en luchar por la reinterpretación de esos conceptos. Y es que la hegemonía, por medio de representaciones e ideas colectivas sobre el trabajo, la vida, la comunidad, etc. articula la diferencia colonial (Castro-Gómez y Grosfoguel, 2007) de formas de gubernamentalidad que actúan precisamente con base en una "otherizacion» y subalternización, «reproduciendo de diferentes maneras la búsqueda por 'civilizar/incluir' a un sujeto que se construye como símbolo de la alteridad, y que suele ser considerado como débil y carente de conocimientos» (Vargas-Monroy y Pujal i Llombart, 2013: 1264).

De este modo, el primer paso consiste en deconstruir las categorías, las creencias y los valores que actúan como pilares de esa cultura excluyente y diferenciadora que es la hegemonía cultural. La historia de las mujeres, como también la de las personas homosexuales, esclavas, inmigrantes, negras $y$, en definitiva, marginadas es la historia de individuos $y$ colectivos víctimas de un sistema opresor, que emplea las ideas para atacar, aislar y someter las identidades que cuestionan el orden hegemónico (Peres Díaz, 2015). Un primer punto de ruptura con esa hegemonía ha de ser la transformación de la economía, tal y como defiende Vargas Soler (2009). En efecto, la economía se constituye como una herramienta de control de los recursos y la redistribución de la riqueza, una herramienta que se ha construido sobre

6 Por «hegemonía» se entiende el conjunto de ideas y representaciones (cultura) que constituyen la base de interpretación de la vida y la sociedad, que son altamente compartidas y cuya imposición corresponde a una élite (Sousa Santos, 2010). Así, la emancipación social consistiría en deconstruir esa hegemonía y establecer caminos de liberación de la opresión desde epistemologías elaboradas por los propios sujetos históricamente excluidos y victimizados (Sousa Santos, 2006). 
un modelo capitalista occidental fundamentalmente impregnado por los valores masculinos de dominación y explotación (Herrero, 2012) ${ }^{7}$.

En esa línea, la descolonización del saber y del ser son las condiciones de posibilidad para poder liberar la subjetividad e intersubjetividad del control eurocéntrico y del dominio moderno, colonial y capitalista. Con ello, esas subjetividades descolonizadas y esas otras formas de conocer también «pueden contribuir a la configuración y desarrollo de prácticas económicas no capitalistas o a la liberación de las prácticas económicas del patrón de poder moderno/colonial/capitalista» (Vargas Soler, 2009: 61).

Por lo tanto, debemos superar el paradigma del beneficio capitalista y enfatizar la economía social, «lugarizando la economía» y articulando:

Una matriz heterárquica de poder (en una estructura común) que trasciende lo económico y lo capitalista y en reconocer la existencia de una diversidad de formas económicas (indígenas, campesinas, populares, comunitarias, urbanas, estatales, capitalistas, no capitalistas, etc.) con singularidades históricas y sustantivas a partir de las cuales pueden desarrollarse prácticas económicas alternativas (Vargas Soler, 2009: 62).

Estas nuevas formas de economía pueden coger fuerza desde los feminismos poscoloniales y, al mismo tiempo, esbozar un nuevo paisaje mental a la hora de entender los derechos humanos. En efecto, los derechos humanos, al menos en su versión occidentalista, son expresión de ese sujeto hipostasiado europeo, formalista hasta el extremo, universal y ahistórico. Por eso, el feminismo poscolonial puede contribuir a deconstruir la idea de que nacemos libres e iguales con una serie de derechos inherentes e inalienables; es decir, puede poner de relieve la necesidad de acompañar esa formalidad de los derechos humanos con una propuesta real por la justicia social de los pueblos. Así, se puede apostar por un yo histórico, una subjetividad arraigada en la facticidad de una experiencia histórica de explotación y opresión, una experiencia que, en última instancia, dé lugar a una noción diferente de los derechos humanos.

El caso de las mujeres indígenas es clarividente. Las mujeres indígenas, sus experiencias de marginación por todo el mundo, encarnan en toda su completud el principio dialógico entre lo universal y lo particular, porque su exigencia de reconocimiento, en tanto que sujetos portadores de una cultura e historia específicas, se inscribe en la reivindicación

\footnotetext{
7 La propuesta de Herrero (2012), desde una perspectiva ecofeminista, se basa en tres ejes. En primer lugar, el decrecimiento de la esfera material de la economía, como principio para la sostenibilidad; en segundo lugar, la interdependencia económica, que debe hacer de los pueblos colectivos más solidarios y conscientes de que la labor de sostenimiento de la vida es la base de toda economía; y en tercer lugar, un reparto de la riqueza más equitativo sobre la premisa de que la acumulación de propiedad y capital ha sobrepasado todos los límites.
} 
de las normas comunes de derecho, ciudadanía y justicia (Masson, 2011 : 168). En esa línea, la lucha indígena, su reconocimiento social y político, puede contribuir a poner de manifiesto la tensión entre la aspiración de validez universal de los derechos de la mujer y la necesidad de articular propuestas que respeten la cultura concreta en que cada mujer o grupo de mujeres instala sus experiencias y saberes.

Así, la dignidad humana aparece desde el feminismo poscolonial sustentada sobre unos pilares sólidos, a saber, «las condiciones materiales y vivenciales de las subjetividades oprimidas y explotadas. Esa carencia de justicia implica la toma de conciencia y el primer paso en la lucha por la reparación de la dignidad atacada» (Rodríguez Prieto, 2015: 99). En consecuencia, los derechos humanos son producto de los procesos que la autoconciencia de opresión y explotación de humanos como ellos generan, y no meras declaraciones pomposas basadas en intuiciones universalistas.

En último término, el feminismo poscolonial es un feminismo de la emancipación social y la cultura de paz desde la resignificación de los conceptos y las prácticas hegemónicas (Castillo Herrerra y Mayela Josefina, 2014). La experiencia de las mujeres en América Latina y África ${ }^{8}$ revela la existencia clara de este feminismo dialógico, toda vez que plantea una posición inclusiva, de igualdad en la diferencia, y de sumar voces y experiencias. En ese sentido, un feminismo de tales rasgos constituye una propuesta política necesaria para la acción, pues permite el disenso y la pluralización desde la diferencia, con la meta última de la paz.

Así, la lucha por la paz desde el feminismo poscolonial arranca a partir de un programa de acción en el que «valore y se dé a la mujer espacios de participación en igualdad por tanto, una sociedad comprometida con la cultura de paz requiere trabajar para que exista acceso a la educación, salud, justicia social, equidad de género, derechos humanos, entre otros» (Castillo Herrera y Mayela Josefina, 2015: 49). En esta línea, es menester generar una solidaridad transnacional, bajo términos no universalistas, sino de cooperación a partir de la diferencia, lo cual implica de suyo una producción de conocimientos decoloniales y un reconocimiento de la legitimidad de otras formas de pensar el feminismo. Estos conocimientos han de conducirnos inexorablemente hacia nuevas visiones de reapropiación de la lógica del poder colonialpatriarcal. Se trata «no de (re)construir lógicas de poder hegemónicas, monolíticas y subjetivas, sino de construir políticas feministas intersubjetivas y pluralistas» (Cuesta, 2016: 65).

En ese sentido, los aportes del feminismo poscolonial sirven no solo para articular propuestas de cara a la emancipación de las mujeres en África o América Latina, sino, también, para abrir el marco de reflexión en torno al feminismo. Así, paradójicamente, el 
feminismo poscolonial, en su lucha contra el feminismo hegemónico, ofrece herramientas para que el propio feminismo hegemónico pueda ser reformulado.

\section{Conclusiones}

El objetivo principal del artículo residía en analizar las líneas maestras del feminismo poscolonial en un intento de deconstrucción del pensamiento feminista occidental. De este modo, hemos podido realizar una exposición de los principales avances teórico-epistémicos del feminismo poscolonial, mostrando su potencial crítico y vertebrando con ello una crítica del etnocentrismo, tanto metodológico como conceptual, del feminismo occidental. A este respecto, hemos podido constatar cómo el feminismo poscolonial reviste de una especial intensidad interna de discusión, variedad y propuestas.

En línea con ello, se han visto también las posibilidades que ofrece el feminismo poscolonial para resignificar, más allá de las categorías de sexo o género, conceptos como el de raza, clase o cómo poder dar algunas líneas para articular formas diferentes de entender la economía o la lucha por la emancipación social. En última instancia, la dualidad entre poscolonialismo y poscolonialidad, esto es, la divergencia entre la historia posterior a la descolonización y la permanencia del trasfondo colonial en forma de legado cognoscitivo, permite poner de relieve la existencia de un poder opresor a escala social y epistémica, de suerte que la primera tarea de las mujeres feministas del Sur ha de consistir en una reapropiación de sus propias experiencias, realidades y saberes, deconstruyendo así la hegemonía y eliminado la «otredad» occidental en que son tematizadas.

Como conclusión final, tan solo recordar al lector o lectora que el presente trabajo no es ni puede, por mor de la verdad, ser exhaustivo, sino constituir, a lo sumo, una aproximación teórica al feminismo poscolonial y algunas de sus claves. Por todo ello, las investigaciones futuras sobre esta cuestión deberán incardinarse en un espectro académico-social más amplio, De forma que se pueda dibujar un mapa de mayor alcance que el que, muy modestamente, se ha expuesto aquí.

\section{Referencias bibliográficas}

ArReAZA, Catalina y TIKCNER, Arlene (2002): «Postmodernismo, postcolonialismo y feminismo: manual para (in)expertos», Colombia Internacional. $N^{\circ}$ 54, pp. 14-38.

BANERJEE, Ishita (2014): «Mundos convergentes: género, subalternidad, poscolonialismo», La ventana. $\mathrm{N}^{\circ} 39$, pp. 7-38. 
BraH, Avtar (1992): «Difference, Diversity and Differentiation», en DonALD, James y All, Rattansi, Race, Culture and Difference. London: Sage, 126-145.

CAstillo herrera, Beverly y MAYela Josefina, Vílchez (2014): «Del feminismo postcolonial al dialógico. Implicaciones para construir una cultura de paz», Revista Científica de FAREM-Estelí. Medio ambiente, tecnología y desarrollo humano. № 12, pp. 42-51.

CAstro-Gómez, Santiago y Grosfoguel, Ramón (eds.) (2007): El giro decolonial: reflexiones para una diversidad epistémica más allá del capitalismo global, Bogotá, Siglo del Hombre.

CUESTA, Marta (2016): «Solidaridad transnacional: Narrativa feminista», Interpretextos. $\mathrm{N}^{\circ}$ 15 , pp. 49-66.

Curiel, Ochy (2007): «Crítica poscolonial desde las prácticas políticas del feminismo antirracista», Nómadas. $\mathrm{N}^{\circ} 26, \mathrm{pp}$. 92-101.

DAvIS, Angela (2005): Mujeres, raza y clase, Madrid, Akal.

De Miguel Álvarez, Ana (2011): «Los feminismos a través de la historia», Mujeres en Red, disponible en <http://www.mujeresenred.net/anademiguel.html> [Consultado 28/01/2017]

Foucault, Michel (1976): Historia de la sexualidad (Vol. I), México, Siglo XXI, 1998.

Herrero, Yayo (2012): «Propuestas ecofeministas para un sistema cargado de deudas», Revista de Economía Crítica. №13, pp. 30-54.

HOOKS, bell (2004): «Mujeres Negras: Dar forma a la teoría feminista», en Otras inapropiables. Madrid, Traficantes de Sueños, 1984.

IVANNA GIGENA, Andrea (2011): «Lecturas poscoloniales y decoloniales de la analítica foucaultiana para el análisis en contextos de herencia colonial», CONFLUENZE. Revista di studi iberoamericani. Vol. 3, N. 2, pp. 1-21.

JABARDO VelASCO, Mercedes (2008): «Desde el feminismo negro, una mirada al género y la inmigración», en Suárez Navas, Martín Díaz y Hernández Castillo (coord.), Feminismos en la antropología: nuevas propuestas críticas, pp. 39-54.

Laura Segato, Rita (2011): "Género y colonialidad: en busca de claves de lectura y de un vocabulario estratégico descolonial», en BIDASECA, Karina y VÁZQUEZ LABA, Vanesa (Comps.), Feminismos y poscolonialidad. Descolonizando el feminismo desde América Latina, pp. 17-49.

LIMIC, Tijana (2015): «Para un diálogo interepistémico y decolonial entre feministas occidentales y no occidentales», Tabula Rasa. № 23, pp. 133-156.

López Souto, África (1999): «Miradas feministas sobre Foucault», Ágora. Papeles de Filosofía. Vol. 18, N¹, pp. 87-105. 
Lugones, María (2008): «Colonialidad y género», Tabula Rasa. № 9, pp. 73-101.

LUGONES, María (2011): «Hacia un humanismo descolonial», La manzana de la discordia. Vol. 6, No 2, pp. 105-119.

Martínez Martín, Irene (2015): «Para repensar la educación en clave feminista y postcolonial frente a la invisibilización de las mujeres y las niñas en la educación africana», Revista de Educación Social. N² 21 , pp. 154-169.

Masson, Sabine (2011): «Sexo/género, clase, raza: feminismo descolonial frente a la globalización. Reflexiones inspiradas a partir de la lucha de las mujeres indígenas en Chiapas», Andamios. Revista de Investigación Social. Vol. 8, № 17, pp. 145-177.

Medina Martín, Rocío (2013): «Feminismos periféricos, feminismos-otros: Una genealogía feminista decolonial por reivindicar», Revista Internacional de Pensamiento Político. Vol. 8, pp. 53-79.

Medina Martín, Rocío (2014): «Resignificaciones conceptuales y epistemológicas en el pensamiento político feminista eurocéntrico desde los feminismos periféricos», Cuadernos Electrónicos de Filosofía del Derecho. № 29, pp. 72-98.

Mekgwe, Pinkie, (2006): «Theorizing African Feminism(s). The 'Colonial' Question», Quest. An African Journa of Philosophy. Vol. XX, N 1-2, pp. 11-22.

MIGNOLO, Walter (1995): «Occidentalización, imperialismo, globalización: Herencias poscoloniales y teorías postcoloniales», Revista lberoamericana. Vol. LXI (170-171), pp. 27-40.

MohantY, Chandra (2008): «Bajos los ojos de Occidente. Academia Feminista y discurso colonial», SuÁrez NAVAZ, Liliana y Hernández, Aída (editoras) Descolonizando el Feminismo: Teorías y Prácticas desde los Márgenes, ed. Cátedra, Madrid, 1-23.

Nnaemeka, Obioma, (2004): «Nego-Feminism: Theorizing, Practicing, and Pruning Africa's Way», Signs. Vol. 29, № 2, pp. 357-385.

Obianuju acholonu, Catherine (1995): Motherism. The Afrocentric Alternative to Feminism, Owerri, Afa Publications.

OgundiPE-LESLIE, Molara, (1994): Re-creating Ourselves: African women and Critical Transformations, Trenton (Nueva Jersey) y Asmara, AfricaWorld Press.

OYEWUMl, Oyeronke (2002): "Conceptualizing Gender: The Eurocentric Foundations of Feminist Concepts and the Challenge of African Epistemologies», Jenda: A Journal of Culture and African Women Studies. Vol. 2, No 1. Disponible en <https://www. codesria.org/IMG/pdf/OYEWUMI.pdf> [Consultado: 05/01/2017]

Peres Díaz, Daniel (2015): «Subjetividad y legitimación: La construcción contemporánea del cuerpo de las mujeres», en Cabrera Espinosa, Manuel y López Cordero, Juan Antonio (eds. lit.), VII Congreso virtual sobre Historia de las Mujeres, pp. 653-664. 
Peres Díaz, Daniel (2016): «Los límites de lo humano: Teoría queer, ciberfeminismo y ontología Cyborg», Tales. Revista de Filosofía. № 6, pp. 151-162.

PÉREZ FlORES, Larisa (2014): «Cuerpos y desplazamientos. Retos feministas en un marco pos/ descolonial», Cuadernos Intercambio sobre Centroamérica y el Caribe, vol. 12, n 1, pp. 97-1 15.

QUIJANO, Aníbal (2005): «Colonialidad del poder, eurocentrismo y América Latina», LANDER y Santiago CASTRO-GómEZ (editores) La colonialidad del saber. Eurocentrismo y ciencias sociales; perspectivas latinoamericanas. 2. ed., 1. reimpr. Buenos Aires: Consejo Latinoamericano de Ciencias Sociales CLACSO [u.a.], pp. 201-246.

Rodríguez Martínez, Pilar (2011): «Feminismos periféricos», Revista Sociedad \& Equidad. N 2, pp. 23-45.

RodríGUez ORTIZ, Roxana (2014): Episetmología de la frontera. Modelos de sociedad y políticas públicas. Kindle Editions.

Rodríguez Prieto, Rafael (2015): «De los márgenes al centro: Una aplicación del feminismo poscolonial a los derechos humanos», Athenea Digital. Vol. 15, № 2, pp. 81-110.

Ron ErRáez, Ximena (2014): «Hacia la desoccidentalización de los feminismos. Un análisis a partir de las perspectivas feministas poscoloniales de Chandra Mohanty, Oyeronke Oyewumi y Aída Hernández», Realis. Revista de estudos antiutilitaristas e Poscoloniais. Vol. 4, N $\mathrm{N}^{\circ}$ 1, pp. 36-60.

Ros, Miquel (2002): «Los valores culturales y el desarrollo económico: una comparación entre teorías culturales», Revista Española de Investigaciones Sociológicas. № 99, pp. 9-33.

Ruiz Trejo, Marisa Gisele y Betemps, Caroline (2015): «Epistemologías y prácticas feministas cruzadas: las posibilidades de la traducción y la importancia decolonial», Relaciones Internacionales. № 27, pp. 169-179.

Sousa SANTOS, Boaventura (2006): Conocer desde el Sur. Para una cultura política emancipatoria. Lima, Fondo Editorial de la Facultad de Ciencias Sociales, UNMSM.

SOUSA SANTOS, Boaventura (2010): Descolonizar el saber, reinventar el poder, Uruguay, Trilce.

SteAdy, Filomena Chioma (ed.)(1981): The BlackWoman Cross-Culturally, An Overview, Cambridge, Schenkman Publishing Co.

Vargas-Monroy, Liliana y Pujal I Llombart, Margot (2013): «Gubernamentalidad, dispositivos de género, raza y trabajo: la conducción de las mujeres trabajadoras», Universitas Psychologica, vol. 12, $\mathrm{n}^{\circ} 4$. ., pp. 1255-1267.

VARGAS SOLER, Juan Carlos (2009): «La perspectiva decolonial y sus posibles contribuciones a la construcción de Otra Economía», Otra Economía. Vol. III, N4, pp. 46-65.

176

Dossiers Feministes, 22, 2017, 157-177 - ISSN: $1139-1219$ - DOI: http://dx.doi.org/10.6035/Dossiers.2017.22.10 
Zirion Aandaluze, Iker e Idagarra Espel, Leire (2015): «Los feminismos africanos. Las mujeres africanas 'en sus propios términos'», Relaciones Internacionales. № 27, pp. 35-54.

Recibido el 27 de febrero de 2017

Aceptado el 5 de mayo de 2017 BIBLID [1 139-1219 (2017) 22: 157-177] 